\title{
Versorgung chronischer Wunden
}

\author{
Care of Chronic Wounds
}

Autor

Institut

\section{Zutt}

Abteilung Dermatologie und Venerologie, Universitätsmedizin Göttingen

\section{Bibliografie}

Dol $10.1055 / \mathrm{s}-0028-1119435$

Akt Dermatol 2009; 35:

90-93 ๑ Georg Thieme

Verlag KG Stuttgart · New York ISSN 0340-2541

Korrespondenzadresse

Dr. med. Markus Zutt

Abteilung Dermatologie

und Venerologie

Universitätsmedizin Göttingen

von-Siebold-Str. 3

37075 Göttingen

mzutt@gwdg.de

\section{Zusammenfassung \\ $\nabla$}

Die moderne Wundversorgung umfasst die kausale Behandlung zugrunde liegender Erkrankungen, chirurgische Verfahren (beispielsweise Wund-Debridement, Venenchirurgie), Lymphdrainage und die korrekte Anwendung geeigneter Wundauflagen. Bei sämtlichen Therapiekonzepten ist es wichtig, auch die Wundränder, die

\section{Einleitung \\ $\nabla$}

Der Hautarzt wird aufgrund der Überalterung unserer Gesellschaft zunehmend mit dem Problem der chronischen Wunde konfrontiert werden. Bereits heute leiden ca. 2 bis 4 Millionen Patienten in Deutschland an chronischen Wunden, davon sind ca. die Hälfte Ulzera der Beine [1]. Dabei nehmen venös bedingte Ulzera einen großen Stellenwert ein. Aufgrund dieser häufigen Erkrankungen werden, nicht zuletzt durch eine unzureichende Wundversorgung, enorm hohe Kosten im Gesundheitssystem produziert. Umso wichtiger erscheint es, heutzutage eine moderne und zielführende sowie kostenorientierte Wundversorgung $\mathrm{zu}$ erlernen und anzubieten. Man kann durch optimale Wundversorgung viele Wunden zur Abheilung bringen. Doch schaut man auf den heutigen Markt von Wundversorgungsprodukten, stellt man sich die Frage nach der optimalen Wundversorgung. Es existieren zahlreiche Firmen, die Wundversorgungsprodukte ohne entsprechende Wirksamkeitsnachweise anbieten. Im Zeitalter von evidenzbasierter Medizin existiert leider nur eine sehr geringe Menge an qualitativ hochwertigen wissenschaftlichen Arbeiten, die sich mit der Wirksamkeit von Wundversorgungsprodukten beschäftigen. Daher erscheint es für den bewussten und optimalen Umgang mit diesen Produkten wichtig, dass sich jeder Dermatologe, der sich mit Wundver- leider oft vernachlässigt werden, geeignet zu behandeln. Hypoallergene Topika tragen dazu bei, Kontaktsensibilisierungen zu vermeiden. In vielen Fällen kann durch die Anwendung der umrissenen multimodalen Therapiekonzepte die Wundheilung initiiert werden. Diese Darstellung gibt einen kurzen Überblick über den gegenwärtigen Stand der Versorgung chronischer Wunden.

sorgung beschäftigt, einen roten Faden seiner persönlichen Wundversorgung mit entsprechend wirksamen, aber auch kostenbewussten Produkten erstellt.

\section{Wundheilung und Auswahl geeigneter Wundauflagen \\ $\nabla$}

Der Prozess der normalen Wundheilung wird in seinem stadienhaften Ablauf durch zahlreiche molekulare und zelluläre Steuerungsmechanismen kontrolliert und moduliert. Es existiert ein komplexes Netzwerk von Zytokinen und extrazellulären Matrixmolekülen (z.B. VEGF, TGF- $\beta$ usw.), die eine normale Wundheilung gewährleisten. $\mathrm{Zu}$ chronischen Wunden kommt es, wenn die Wundheilungsphasen durch Störungen in diesem komplizierten molekularen Netzwerk fehlerhaft ablaufen. Viele chronische Wunden persistieren in der Entzündungsphase, häufig sind Proteasen, z.B. Metalloproteinasen, im Wundsekret erhöht oder es sind von Bakterien freigesetzte proteolytische Enzyme überschüssig vorhanden, die eine Wundheilung inhibieren [2]. Die moderne Wundversorgung versucht, in diese fehlerhaft ablaufenden Wundheilungsphasen einzugreifen, z. B. durch die Kontrolle von schädigenden Proteasen. Neben der Behandlung der zugrunde liegenden Erkrankung (z.B. Beseitigung des venösen Refluxes bei chronisch-venösen 


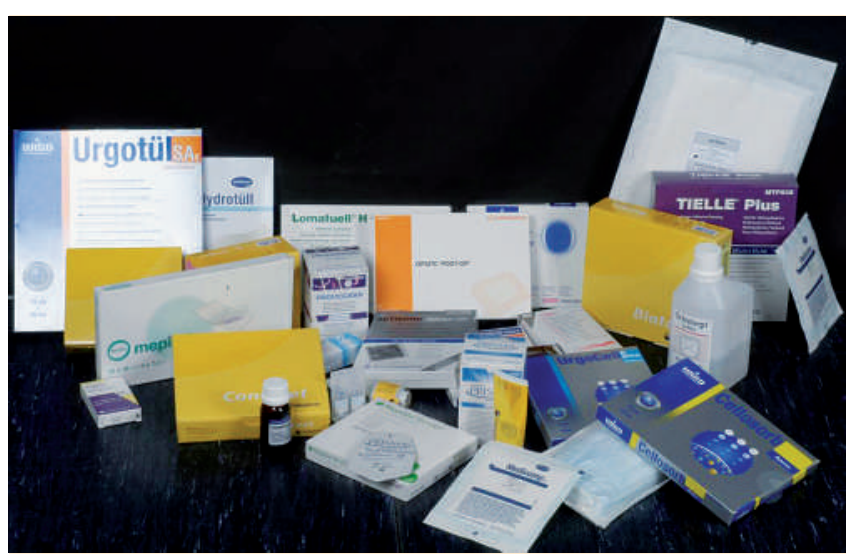

Abb. 1 Wundversorgungsprodukte einer dermatologischen Normalstation.

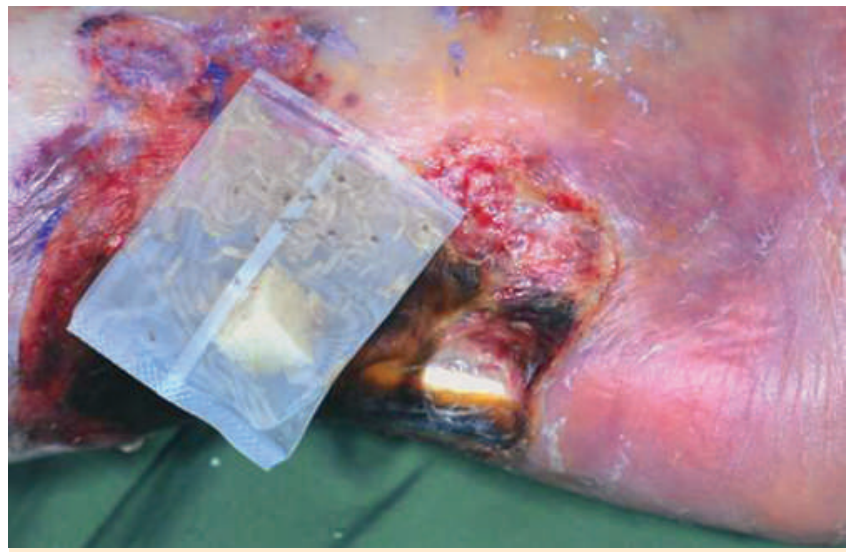

Abb. 2 Madentherapie bei einem nekrotisch belegten Ulcus cruris.

Wunden durch operative Sanierung oder Kompressionstherapie, Einstellung des Diabetes bei diabetischen Ulzera oder Verbesserung der arteriellen Durchblutungssituation bei arteriell bedingten Ulzera) erscheint für die Wahl des geeigneten Verbandstoffes zum einen die Wundbeurteilung selbst, aber auch die Gesamtbeurteilung des Patienten wichtig zu sein ( $\bullet$ Abb. 1). Ein Wundverband muss praktikabel einsetzbar und auch entsprechend den häuslichen Umgebungen für den Patienten anwendbar sein. Zur Wundbeurteilung gehören Wundlage, Wundgrund, Wundexsudat und - nicht zuletzt - die Wundumgebung. Für den Dermatologen erscheint eine adäquate Versorgung des Wundrandes ebenso wichtig, wie die Versorgung der Wunde selbst. Dabei sei auf das große Problem der Kontaktsensibilisierungen hingewiesen. Voraussetzung für eine Wundheilung ist ein entsprechend gesäuberter Wundgrund. Hierfür erscheint ein chirurgisches Debridement unerlässlich. Eine weitere hervorragende Möglichkeit zur Wundsäuberung stellt die biochirurgische Therapie mit Lucillia sericata dar ( $\bullet$ Abb. 2). Enzymatische Wundreinigungsprodukte sind bei fraglicher Wirksamkeit in Einzelfällen indiziert.

\section{Bakterielle Besiedelung und Antisepsis \\ $\nabla$}

Die Rolle der bakteriellen Besiedelung von chronischen Wunden wird kontrovers diskutiert. Sicher benötigen nicht alle chronischen Wunden eine antiseptische Behandlung, denn durch eine bakterielle Besiedelung ist die Wundheilung nicht zwingend beeinträchtigt. Die Indikationen für eine antiseptische Wundtherapie sind klinisch infizierte Wunden oder infektionsgefährdete Wunden. Dabei sollten Antiseptika den Anforderungen eines breiten Wirkspektrums bei guter Gewebeverträglichkeit und Farblosigkeit entsprechen. Dies ist für die modernen Antiseptika wie Polyhexanid und Octenidin gegeben.

\section{Wundauflagen}

$\nabla$

Bei Wundauflagen sollte man neben einfacher Handhabung, der Möglichkeit von großen Verbandwechselintervallen und damit Kosteneinsparungen, auf die Eigenschaften der verschiedenen Wundauflagen achten. Hydrokolloide gehören zur Gruppe der Verbandstoffe mit mäßigen Flüssigkeitsaufnahmen und sind heutzutage nur noch bei oberflächlichen chronischen Wunden geeignet. Den Hydrokolloiden vorzuziehen sind in vielen Fällen Schaumverbände, die aus einem Polyurethan-Schaumstoff mit einer semipermeablen Deckschicht bestehen. Diese erhalten das feuchte Wundmilieu. Das Prinzip der feuchten Wundheilung hat das jahrelang existierende Prinzip der trockenen Wundheilung und der Bevorzugung von Farbstoffen abgelöst. Alginate gehören neben Hydrogelen und Kollagen-Produkten in die Hand eines wundtherapeutisch tätigen Dermatologen. Eine Renaissance hat in letzter Zeit die Anwendung von Silber erfahren. Dieses ist in Form von silberbeschichteten Wundauflagen heutzutage in breiter Anwendung. Allerdings ist die unkritische Applikation von silberbeschichteten Wundauflagen nicht empfehlenswert, denn eine Resistenzentwicklung und die Entstehung von Lücken im bakteriellen Spektrum sind bei prolongierter Anwendung unvermeidbar. Kurzfristig ist die Anwendung silberbeschichteter Wundauflagen bei kritisch infizierten Wunden zu empfehlen. Eine weitere wirksame Wundauflage stellen Aktivkohle-Verbände zur Exsudat-Bindung auf stark geruchsintensiven Wunden dar.

\section{Kontaktsensibilisierungen bei Patienten mit chronischen Wunden \\ $\nabla$}

Bei der Anwendung von Wundauflagen und Externa, insbesondere bei chronischen Wunden und chronischen venösen Ulzera, sei auf die hohen Kontaktallergie-Raten hingewiesen ( $\bullet$ Abb. 3). Dabei sind nach wie vor Salbengrundlagen sowie Antibiotika und Konservierungsstoffe, aber auch z.B. PVP-Jod in hohem Maße vertreten [3]. Immer noch findet man z. B. Wollwachsalkohole in entsprechenden Wundgazeverbänden. Der Gebrauch solcher Produkte sollte grundlegend vermieden werden. Es existieren leider bis heute wenige Daten über Kontaktsensibilisierungen bei Anwendung von modernen Wundtherapeutika. Diese Fragestellung wird in Zukunft von Interesse sein. 


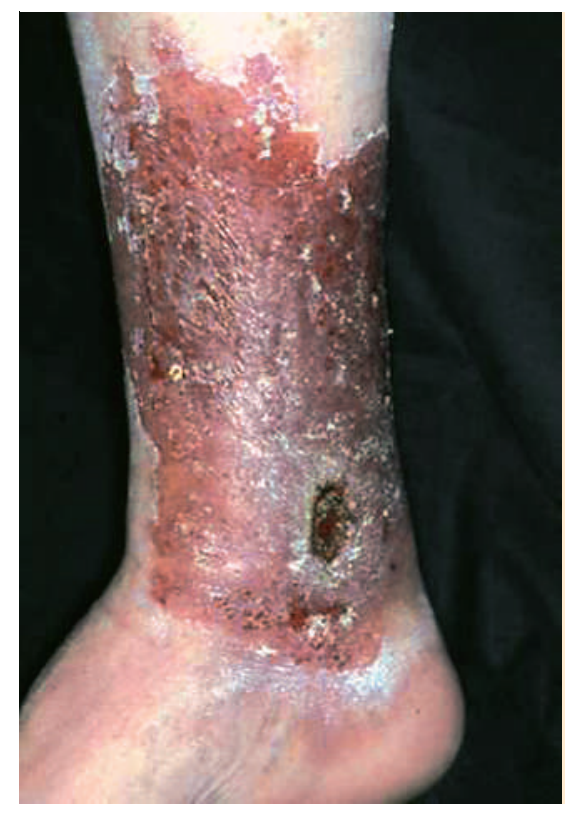

Abb. 3 Ulcus cruris venosum mit allergischem Kontaktekzem.

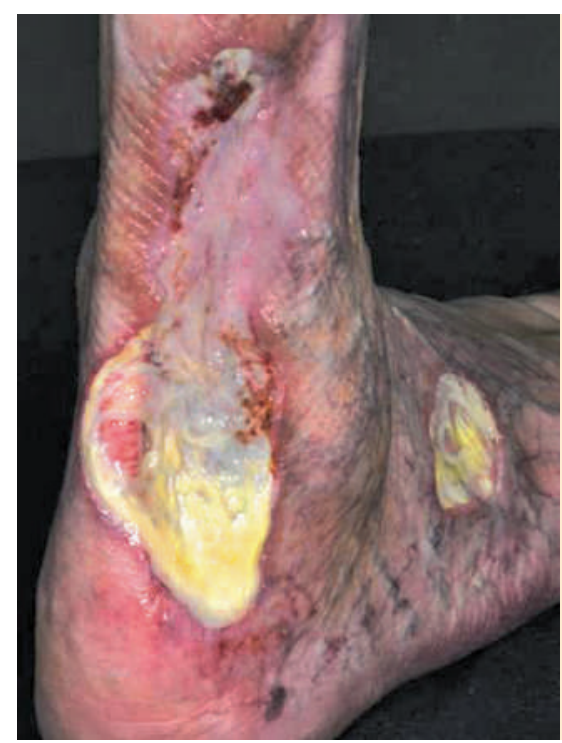

Abb. 5 Vaskulopathisches Ulcus cruris (Pyoderma gangränosum).

\section{Vakuumtherapie}

$\nabla$

Einen großen Stellenwert hat die Durchführung der Vakuumtherapie in der Versorgung von chronischen Wunden ( $\bullet$ Abb. 4). Insbesondere bei Problemwunden wie vaskulitischen oder vaskulopathischen Ulzera ( $\bullet$ Abb. 5 ) ist die Anwendung dieses Verfahrens in Kombination mit entsprechenden Systemtherapien zu empfehlen [4]. Problematisch erscheint nach wie vor, dass die Vakuumtherapie in Deutschland nicht vertragsärztlich zugelassen ist, sodass sich Kostenübernahmeprobleme bei ambulanter Durchführung dieser Therapie ergeben.

\section{Physikalische und Kompressions-Therapie \\ $\nabla$}

Insbesondere zur Behandlung chronisch venöser Ulzera sind neben adäquater Wundversorgung die Durchführung krankengymnastischer Maßnahmen zur Mobilisation des oberen Sprunggelenkes sowie regelmäßige manuelle Lymphdrainagen und die konsequente medizinische Kompressionstherapie uner-

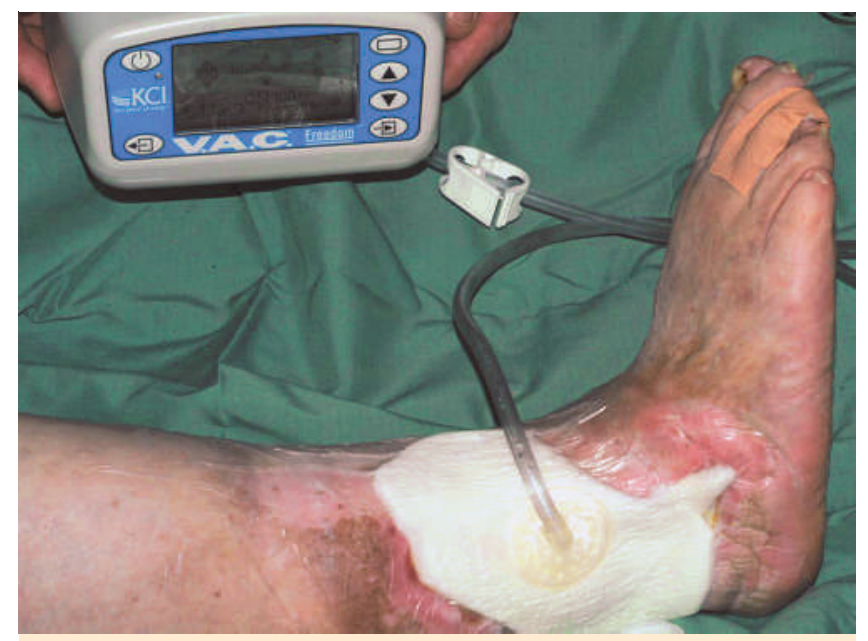

Abb. 4 Anlage einer Vakuumtherapie.

lässlich. Dabei kommen zunehmend moderne Kompressionsstrümpfe, die eigens zur Behandlung von Ulcera crurum entwickelt worden sind, zur Anwendung. Sie lassen sich ideal mit modernen Wundversorgungsprodukten kombinieren und erscheinen im Vergleich zum klassischen phlebologischen Kompressionsverband mit Kurzzugmaterialien überlegen. Es kann ein standardisiertes Anlegen dieser Strümpfe bei konstant gleichbleibendem Druck über 24 Stunden gewährleistet werden [5]. Die Patientenakzeptanz ist, insbesondere bei mobilen Patienten, deutlich höher, als bei klassischen Kurzzugverbänden. Daher ist das Tragen dieser Ulkus-Strümpfe aus phlebologischer und dermatologischer Sicht uneingeschränkt zu empfehlen.

Zusammenfassend besteht die moderne Wundversorgung von chronischen Wunden aus einer Kombination von Behandlung der Grunderkrankung, operativer Sanierung, additiven Behandlungsformen wie Krankengymnastik und Lymphdrainage und der Auswahl des korrekten, stadiengerechten Wundauflagekonzeptes mit entsprechender Wundrandumgebungstherapie. Bei Beachtung dieser Grundsätze ist in vielen Fällen eine erfolgreiche, für den Patienten akzeptable und für den Dermatologen motivierende Therapie einer chronischen Wunde möglich.

\section{Integriertes Konzept der klinischen Wundversorgung \\ $\nabla$}

Wir versuchen in der Hautklinik Göttingen im Rahmen unserer stationären und teilstationären Behandlung den o.g. Grundsätzen der Wundbehandlung zu entsprechen. Es werden in unserer Klinik alle gängigen phlebochirurgischen Operationen zur Beseitigung eines venösen Refluxes (Varizenexhairese mit Crossektomie und Stripping), paratibiale Fasziotomien, Spalthautdeckungen etc. durchgeführt. Eine enge Kooperation mit der Klinik für Gefäßchirurgie zur interdisziplinären Behandlung vorwiegend arteriell verursachter Wunden ist gegeben. Insbesondere die konservative Ulkustherapie wird durch eine enge Vernetzung von ärztlichem und pflegerischem Personal sowie durch eine gute Kooperation mit der physiotherapeutischen Abteilung unserer Klinik optimal gewährleist. So erhält jeder stationäre wie auch teilstationäre Patient bei entsprechender Indikation eine tägliche krankengymnastische Therapie sowie manuelle oder apparative Lymphdrainage in Kombination mit adäquater Kompressionstherapie. Wir nehmen an internationalen Studien zur 
Überprüfung der Wirksamkeit von verschiedenen Wundversorgungsprodukten teil, um so mehr Klarheit in das übergroße Angebot an Wundversorgungsprodukten in Deutschland zu bekommen. Zusammenfassend wird in der Universitäts-Hautklinik Göttingen versucht, dem zunehmenden Problem der chronischen Wunden auf höchstem Niveau gerecht zu werden und die Patientenversorgung optimal zu gewährleisten.

\section{Abstract}

\section{Care of Chronic Wounds}

$\nabla$

Modern care of chronic wounds comprises treatment of the causing disease, surgical procedures (e.g. elimination of refluxive varicose veins, surgical wound debridement), lymphatic drainage, physiotherapy and, in addition, the appropriate application of modern wound dressings. Paying particular attention to the wound margins, which are often neglected, is very impor- tant in modern wound care concepts. Hypoallergenic ointments are recommendable in order to avoid contact allergic reactions. Using this combined concept, it is possible to induce a healing process of the chronic wound. The aim of this paper is to give a brief overview about the state of the art in modern wound care.

\section{Literatur}

1 Tautenhahn J, Bürger T, Lippert $H$. Wounds, wound healing, chronic wounds (part 1). Zentralbl Chir 2003; 128: W2 -W7

2 Eming SA, Kaufmann J, Löhrer R, Krieg T. Chronische Wunde. Hautarzt 2007; 58: 939-944

3 Freise J, Kohaus S, Korber A et al. Contact sensitization in patients with chronic wounds: results of a prospective investigation. JEADV 2008; 22: $1203-1207$

4 Zutt M, Haas E, Krüger $U$ et al. Successful use of vacuum-assisted closure therapy for leg ulcers caused by occluding vasculopathy and inflammatory vascular diseases - a case series. Dermatology 2007; 214: $319-324$

5 Jünger $M$, Sippel $K$. Compression therapy in chronic venous insuffiency. New test procedures and therapeutic options. Hautarzt 2003; 54: $1045-1052$ 